\title{
Blood ammonia and lactate responses to incremental exercise in highly-trained male sprinters and triathletes
}

\author{
Adam Kantanista ${ }^{1}$, Krzysztof Kusy², Ewa Zarębska², Michał Włodarczyk ${ }^{2}$, \\ Monika Ciekot-Sołtysiak², Jacek Zieliński² \\ 1 Department of Didactics of Physical Activity, University of Physical Education, Poznań, Poland; ${ }^{2}$ Department of \\ Athletics, University of Physical Education, Poznań, Poland
}

\section{Summary}

Study aim: To compare the blood ammonia and lactate concentrations in sprinters and triathletes during an incremental treadmill exercise test and in the 30 minutes of recovery.

Material and methods: The study included 10 male sprinters and 14 male triathletes who compete at the national and international level. A treadmill test until exhaustion was administered. Blood samples for ammonia and lactate were obtained when the athletes were at rest, during and immediately after exercise, and between 5 and $30 \mathrm{~min}$ after exercise.

Results: The ammonia concentration and time course were similar in the sprinters and triathletes $\left(\mathrm{F}=1.81, \mathrm{p} \geq 0.05, \eta^{2}=0.08\right)$. An exercise-related increase in blood ammonia was almost linear, regardless of the exercise intensity. In the case of lactate, the interactions between the concentrations measured in the sprinters and triathletes were statistically significant $(\mathrm{F}=5.78$, $\left.\mathrm{p} \leq 0.001, \eta^{2}=0.21\right)$. Post-hoc tests revealed that the lactate concentrations differed significantly between the sprinters and triathletes in the 18 th $\min (\mathrm{p} \leq 0.01)$ and the $21 \mathrm{st} \min (\mathrm{p} \leq 0.001)$ of the exercise test. The blood lactate increased in a nonlinear manner (slowly at lower intensities and rapidly at higher intensities). During the 30 min recovery period, both the ammonia and lactate levels decreased linearly. However, in the sprinters, the peak values were maintained in the first stage of recovery (5 min post-exercise).

Conclusions: The study showed that the blood ammonia concentration may be a useful marker of exercise-related metabolic responses in sprint-trained as well as in endurance-trained competitive athletes. Blood ammonia levels were more intensitysensitive across the whole intensity range during the incremental exercise when compared to the blood lactate levels.

\section{Key words: Blood ammonia - Blood lactate - Treadmill test - Sprinters - Triathletes}

\section{Introduction}

The knowledge of an individual's response to exercise in highly-trained athletes enables the effective implementation of training loads. The concentrations of muscle adenosine triphosphate (ATP) metabolites, which are directly influenced by the intensity, duration and type of exercise, can serve as biomarkers for energetic stress and muscle fatigue. The most well-known of these biomarkers include lactate, ammonia and oxypurines [10]. Lactate is produced in the cytosol in the glycolytic pathway, through the reduction of pyruvate and the oxidation of reduced nicotinamide adenine dinucleotide $(\mathrm{NADH})$ to $\mathrm{NAD}^{+}$. This reaction is catalysed by lactate dehydrogenase [24]. During exercise, the level of blood lactate increases when the phosphate sources, derived mainly from phosphocreatine
(PCr), are reduced. If the lactate production exceeds its clearance, lactic acidosis will progress [10]. Also, when the levels of hydrogen ions $\left(\mathrm{H}^{+}\right)$and NADH increase, they inhibit phosphofructokinase activity, which leads to reduced glycogenolysis [19]. Ferri et al. [9] suggested that highly-trained athletes can produce and tolerate higher lactate concentrations during incremental exercise until exhaustion.

Recently, purine derivatives have proved to be effective markers for energetic stress, training status and performance in highly trained athletes, regardless of their sport specialisation [29, 30, 31, 32]. However, hypoxanthine (Hx), xanthine $(\mathrm{X})$ and uric acid (UA) represent the final stage of ATP metabolism and accumulate relatively late in the blood. Ammonia is the direct product of adenosine monophosphate (AMP) degradation, which occurs earlier in time [30]. Thus, ammonia may be an interesting 
biomarker for monitoring exercise and training. Furthermore, Yuan et al. [28] suggested that ammonia could be a more important indicator than lactate, when used in sport training.

The exercise-induced mechanism of ammonia production can be briefly described as follows. When the consumption of ATP exceeds the ATP supply, the ATP/ADP (adenosine diphosphate) ratio decreases. The enzyme adenylate kinase then transfers one energy-rich phosphate group from one ADP molecule to another, resulting in the formation of one ATP and one AMP molecule. Then the AMP is deaminated by the AMP deaminase to inosine monophosphate (IMP) and ammonia [14]. During highintensity exercise, the deamination of AMP is the main source of ammonia, as is the catabolism of branched amino acids (leucine, isoleucine and valine) during prolonged submaximal exercise [23, 26, 27].

Gorostiaga et al. [11] observed a significant correlation between the blood ammonia level and the muscle ATP, and concluded that blood ammonia could be used as an extracellular marker for muscle ATP content. Moreover, ammonia affects both the central nervous system and the muscle functioning $[16,27]$; thus, it is also considered as a biomarker for peripheral muscle fatigue [10]. Exerciseinduced hyperammonemia may influence brain function, mainly during exhausting exercise [27]. Nybo et al. [17] also suggested that the cerebral uptake and the accumulation of ammonia during prolonged exercise may provoke fatigue.

Previous research has mainly focused on changes in the blood ammonia and lactate concentrations in response to repeated resistance exercise [11] and to prolonged constant moderate-intensity exercise $[18,21]$ in physically active healthy subjects, but not in competitive athletes. Reports concerning the ammonia response in highly-trained athletes during incremental exercise until exhaustion and during recovery are lacking. Therefore, the aim of this study was to compare the blood ammonia and lactate concentrations in sprinters and triathletes during an incremental treadmill exercise test and in the 30 minutes of recovery. We also tried to evaluate and compare the potential usefulness of these both markers in training control.

\section{Material and methods}

\section{Participants}

The study included 10 male sprinters who specialise in the $100 \mathrm{~m}, 200 \mathrm{~m}$ and $4 \times 100 \mathrm{~m}$ relay events, and compete at the national and international level. This first group was aged $23.8 \pm 4.1$ years, with a competitive sport training background ranging from 3 to 14 years, weight of $84.2 \pm 5.5 \mathrm{~kg}$, height of $186.0 \pm 4.8 \mathrm{~cm}$, and maximal oxygen consumption $\left(\mathrm{VO}_{2 \max }\right)$ of $50.7 \pm 3.3 \mathrm{ml} / \mathrm{kg} / \mathrm{min}$.
The second group consisted of 14 male triathletes who also compete at the national and international level, and were aged $24.1 \pm 4.3$ years, with a competitive sport training background of 4 to 12 years, weight of $73.4 \pm 6.2 \mathrm{~kg}$, height of $181.3 \pm 6.2 \mathrm{~cm}$, and $\mathrm{VO}_{2 \max }$ of $66.8 \pm 3.2 \mathrm{ml} / \mathrm{kg} /$ min. The study was performed during the final stage of the preparation phase of a one-year training cycle.

The athletes were informed of the procedures and gave their written consent to participate in the study. The protocol of the study was approved by the Local Bioethical Committee at the Poznan University of Medical Sciences.

\section{Incremental treadmill test protocol}

An incremental treadmill (Pulsar, h/p/cosmos, Germany) test until exhaustion was administered in the morning. The test was completed in the Human Kinetics Laboratory "LaBthletics" at the Poznan University of Physical Education about $3 \mathrm{~h}$ after the participants had consumed a light breakfast (bread and butter, water, without coffee or tea). After 3 minutes of standing on the treadmill, the athletes started the exercise protocol at a speed of $4 \mathrm{~km} / \mathrm{h}$. After 3 minutes, the speed was increased to $8 \mathrm{~km} / \mathrm{h}$. From this point on, the speed was increased by $2 \mathrm{~km} / \mathrm{h}$ after every 3 minutes, with 30 second intervals between the stages which were needed to collect the blood samples. The participants continued running until they reached volitional exhaustion. The athletes were verbally encouraged to continue for as long as possible. Once exhaustion (maximal speed) was reached, the athletes underwent a 30 minute recovery period, during which blood samples were collected after 5, 10, 15, 20 and 30 minutes (Fig. 1).

\section{Blood ammonia and lactate measurement}

Blood lactate and ammonia samples were obtained when the athletes were at rest, during and immediately after exercise, and between 5 and $30 \mathrm{~min}$ after exercise (Fig. 1). The blood samples were obtained from the antecubital vein into S-Monovette (2.7 ml, Sarstedt, Germany) with lithium heparin as an anticoagulant, via a peripheral venous catheter (dimensions $1.3 \times 32 \mathrm{~mm}$, BD Venflon Pro, Sweden). Throughout the duration of the test, the athletes were given an intravenous drip with a saline solution $(0.9 \% \mathrm{NaCl})$ administered by a health care professional to prevent blood coagulation in the catheter. The samples were immediately analysed. To determine the concentration of blood ammonia, $20 \mu \mathrm{l}$ of whole blood was applied on a test strip and analysed using a PocketChem BA device (Arkray, Japan). A Biosen C-line analyser (EKF Diagnostics, Germany) was used to measure the blood lactate accumulation. In brief, $20 \mu \mathrm{l}$ of whole blood was added into the prefilled test tube using a capillary tube. The L-lactate contained in the sample was then converted enzymatically to pyruvate and hydrogen peroxide, which was detected by the electrode. 


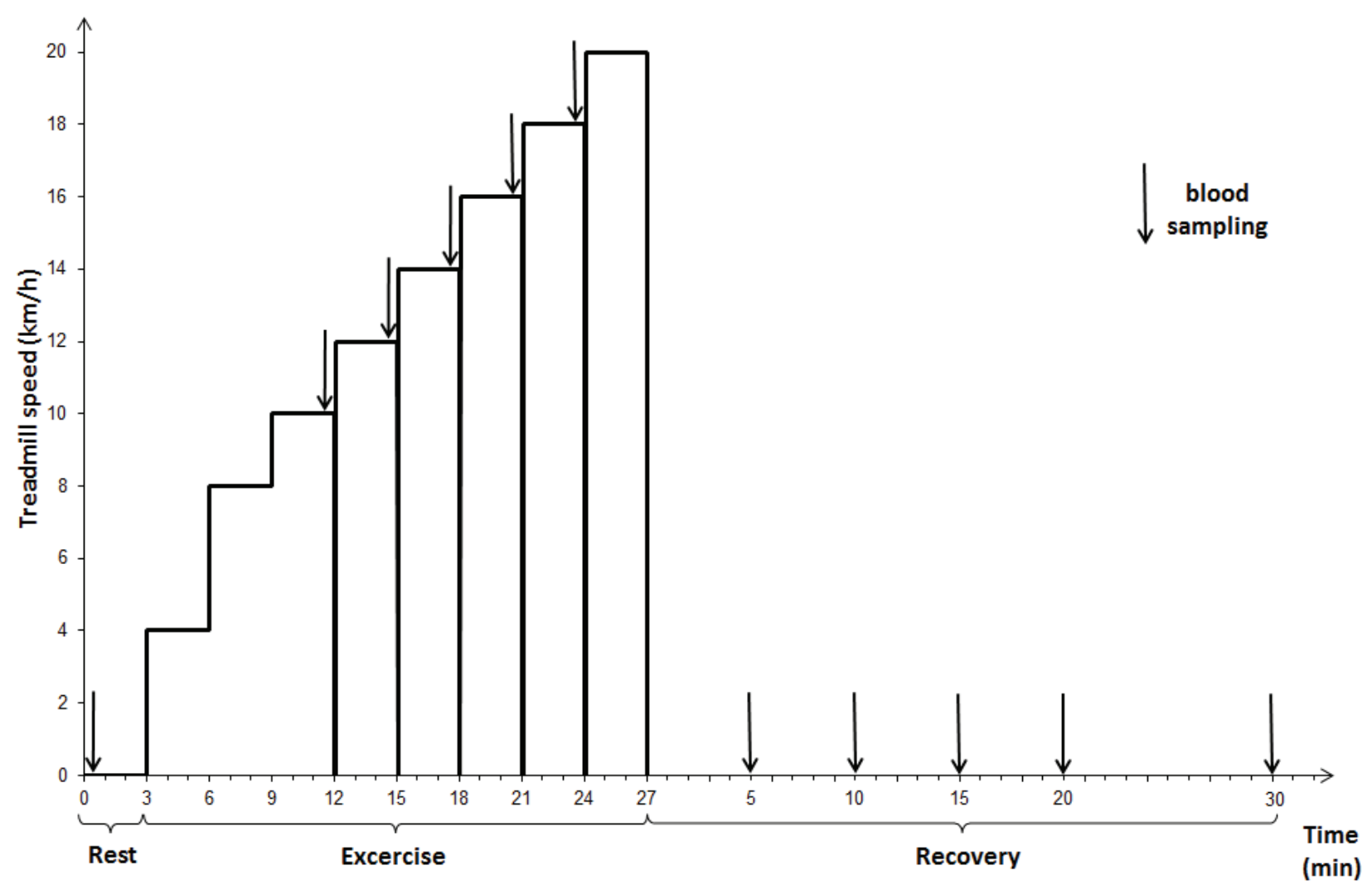

Fig. 1. The maximal graded exercise test protocol and recovery period. Arrows indicate the blood sampling intervals

\section{Statistical analysis}

A two-way ANOVA for repeated measures was used to analyse the differences in blood ammonia and lactate levels between the groups of sprinters and triathletes, and between the measurements at established time intervals during the periods of exercise and recovery. The Tukey's HSD post-hoc tests were performed to assess the significance of the differences between pairs of measurements, while the Eta-squared $\left(\eta^{2}\right)$ was calculated to determine the effect size. If the interaction between the groups was non-significant, the analysis of differences between consecutive measurements was performed in the combined group of sprinters and triathletes. The significance level was set at $\mathrm{p} \leq 0.05$, and the statistical analysis was carried out using STATISTICA 10.0 software (StatSoft, Tulsa, OK).

\section{Results}

The main results are illustrated in Figure 2. In the case of ammonia, the interactions between concentrations measured in the sprinters and triathletes were non-significant $\left(F=1.81, p \geq 0.05, \eta^{2}=0.08\right)$, i.e. the groups did not significantly differ with regards to the pattern of the ammonia time course. Also, post-hoc tests revealed that there were no significant differences in ammonia concentrations between corresponding pairs of measurements (sprinters vs triathletes) at any exercise stage, despite the continued slightly greater values in the sprinters. Statistically significant differences between subsequent ammonia concentrations (in the combined group of sprinters and triathletes) were observed between the $12^{\text {th }}$ min and $15^{\text {th }}$ min $(p \leq 0.01)$, between the $15^{\text {th }} \min$ and $18^{\text {th }} \min (p \leq 0.001)$, between the $18^{\text {th }} \min$ and $21^{\text {st }} \min (\mathrm{p} \leq 0.001)$, and between the $21^{\text {st }}$ min and the final stage $(p \leq 0.001)$ of the exercise test. All of the differences in ammonia concentrations measured during the recovery period were statistically significant.

During the incremental exercise test, the highest concentrations of ammonia were found to be $69.00 \pm 10.49 \mu \mathrm{mol} / 1$ in the sprinters and $68.00 \pm 7.80 \mu \mathrm{mol} / 1$ in the triathletes, and were observed at the time of exhaustion in both groups.

In the case of lactate, the interactions between concentrations measured in the sprinters and triathletes were statistically significant $\left(F=5.78, p \leq 0.001, \eta^{2}=0.21\right)$. Thus, the patterns of the time course for lactate were different for these two groups. The post-hoc tests revealed that lactate concentrations differed significantly between the sprinters and triathletes in the 18 th $\min (\mathrm{p} \leq 0.01)$ and the $21 \mathrm{st}$ min $(p \leq 0.001)$ of the exercise test (Fig. 2).

In the sprinters, statistically significant differences in the lactate concentrations were observed between the start of the exercise and the $15^{\text {th }} \min (p \leq 0.01)$, between 


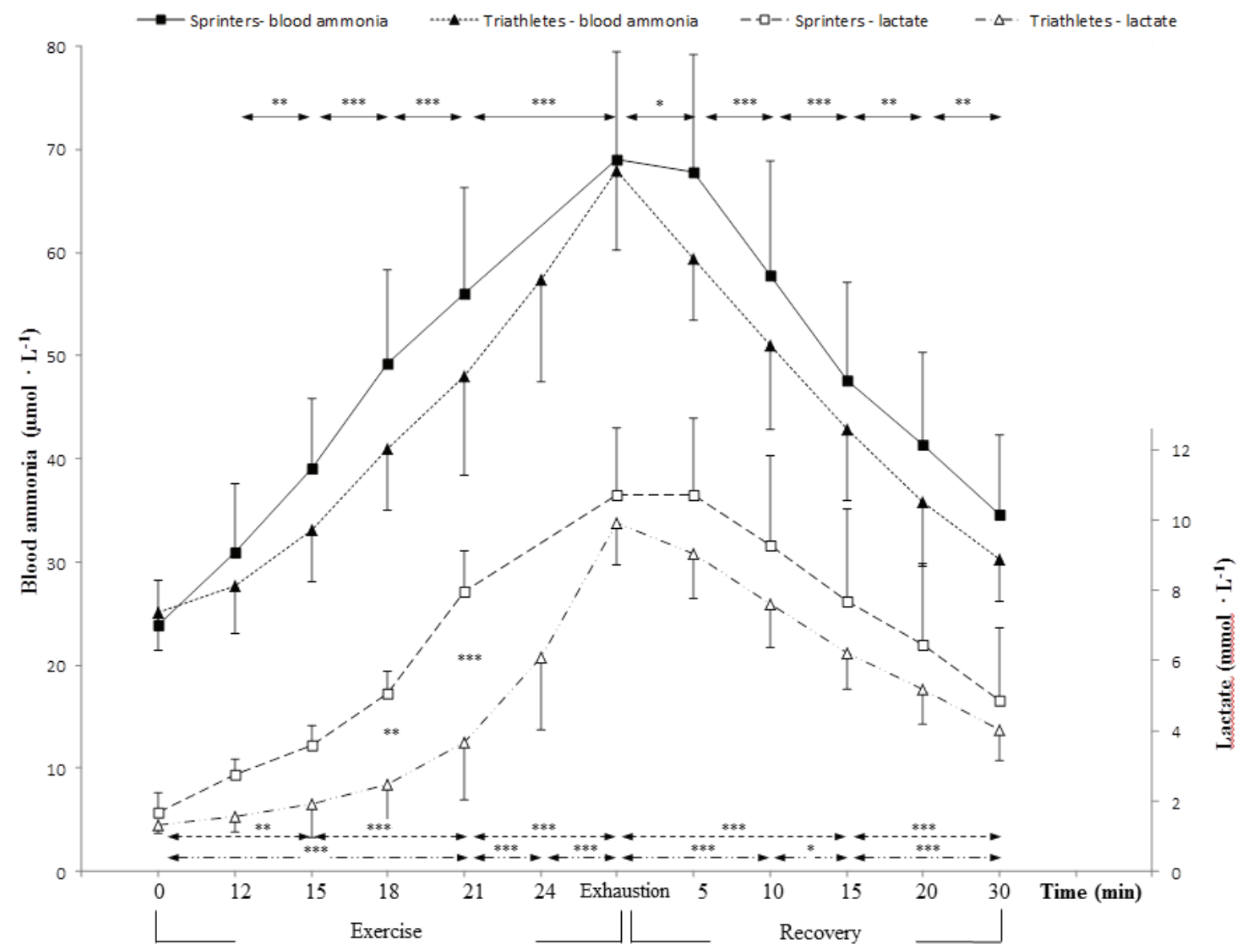

Fig. 2. Blood ammonia and lactate concentrations during incremental treadmill test until exhaustion and post-exercise recovery in sprinters and triathletes. Arrows indicate differences in blood ammonia and lactate concentrations between blood samples taken at subsequent time intervals. Significant differences: $* * * \mathrm{p} \leq 0.001, * * \mathrm{p} \leq 0.01, * \mathrm{p} \leq 0.05$

the $15^{\text {th }} \min$ and $21^{\text {st }} \min (\mathrm{p} \leq 0.001)$, and between the $21^{\text {st }}$ min and the time of maximal speed $(\mathrm{p} \leq 0.001)$. Statistically significant differences were also observed between the time of exhaustion and the $15^{\text {th }} \min (p \leq 0.01)$ of the recovery period, and between the $15^{\text {th }}$ and $30^{\text {th }}$ min of the recovery period.

In the triathletes, statistically significant differences in the lactate concentrations were observed between the start of the exercise and the $21^{\text {st }} \min (p \leq 0.001)$, between the $21^{\text {st }} \min$ and $24^{\text {th }} \min (\mathrm{p} \leq 0.001)$, and between the $24^{\text {th }}$ min and the time of exhaustion $(\mathrm{p} \leq 0.001)$. Statistically significant differences were also observed between the time of the termination of the exercise and the $10^{\text {th }} \mathrm{min}$ ( $p \leq 0.01)$ of the recovery period, as well as between the $10^{\text {th }}$ and $15^{\text {th }}$ min and between the $15^{\text {th }}$ and $30^{\text {th }}$ min of the recovery period.

During the incremental exercise test, the highest concentration of lactate in the sprinters was found to be $10.43 \pm 2.17 \mathrm{mmol} / 1$ (at the $5^{\text {th }} \mathrm{min}$ of the recovery period). In the triathletes, the highest concentration of lactate was $9.61 \pm 1.19 \mathrm{mmol} / \mathrm{l}$, which was observed at the time of exhaustion (Fig. 2).

\section{Discussion}

Our results show the changes in blood ammonia and lactate concentrations in highly-trained sprinters and triathletes, in response to an incremental treadmill exercise. The main observations of this study are that: (i) the patterns of the time course and the values of the blood ammonia concentrations are similar, regardless of the athletes' training adaptation (speed-power versus endurance), whereas there are considerable differences regarding the changes in blood lactate concentrations; and (ii) the blood ammonia concentration changes significantly in an almost linear manner from the start of the exercise until the time of exhaustion, whereas there is a nonsignificant change in the lactate concentration at early (low-intensity) exercise stages, followed by a rapid increase in this concentration at higher intensities. These similarities and differences are, in general, related to the distinct metabolic pathways that lead to ammonia and lactate accumulation in the blood. In practical terms, the obtained picture of changes may and should imply important consequences for using both of 
these biochemical markers in exercise and training control for competitive sport. Below, we explain the main metabolic mechanisms and suggest potential practical implications of these findings.

\section{Training specificity}

Our athletes represent two different training models. Sprint training is mainly based on high-intensity exercise, during which the majority of the energy for muscular activity comes from anaerobic sources [7, 25]. In male sprinters, the contribution of anaerobic energy systems during a 100 $\mathrm{m}$ track sprint is $89-90 \%$, based on lactate/phosphate creatine ratio, or $75-79 \%$ if calculated from the accumulated oxygen deficit $[6,25]$. In contrast, high-performance endurance training, as has been shown in a number of studies, is mostly based on exercise that is done below the anaerobic threshold [3, 8, 22]. For example, during a track run over a distance of $3000 \mathrm{~m}$ it has been estimated that $\sim 86 \%$ of the metabolic energy supply is derived from aerobic sources and $\sim 14 \%$ from anaerobic energy systems $[6,8]$.

\section{Ammonia during exercise}

In this study, no significant differences between sprinters and triathletes in terms of their concentration of blood ammonia were found, although the triathletes continued their test at speeds of up to $18 \mathrm{~km} / \mathrm{h}$, while the sprinters ran at up to $16 \mathrm{~km} / \mathrm{h}$. This may be explained by the fact that, regardless of the energy system that dominates at any exercise intensity or specific training adaptation, ammonia production mainly reflects the ATP breakdown and the energy status and is linearly proportional to the exercise intensity. The main factors that affect the concentration of blood ammonia during and after exercise are muscle ammonia production, as well as its release from the muscle and/or removal from the blood $[12,20]$. Although ammonia production is stoichiometrically related to adenine nucleotide breakdown during high-intensity exercise, the amino acid catabolism also contributes to ammonia formation during prolonged moderate-intensity exercise [21].

As was mentioned earlier, ammonia is a byproduct of the deamination of AMP to IMP via the AMP deaminase. Dudley and Terjung [5] showed that, in fast-twitch muscle fibres (a higher percentage of which is characteristic for sprinters) the activity of AMP deaminase is lower than in slow-twitch fibres, which results in lower blood ammonia concentrations. However, lower ammonia accumulation in blood has also been observed in endurance athletes (who have a high percentage of slow-twitch fibres) [12]. Therefore, it seems that the performance level, specific training programme and the intensity and mode of exercising may have a strong impact on the blood ammonia concentration. For example, Ravier et al. [20] found differences between international and national level karate athletes in their increase in ammonia concentrations until the peak amount during a supramaximal test. The national karate athletes attained much higher ammonia levels, despite a similar maximal accumulated oxygen deficit. As the authors suggested, these differences could be due to either a higher anaerobic contribution to the energy supply in the national athletes, or a higher removal ability in the international athletes. In our study, both athletic groups had similar time courses and levels of ammonia concentration, which could be related to similar (international) performance levels in their disciplines. We can only speculate that the still-observed slight but non-significant difference between the groups in terms of their blood ammonia concentrations can be attributed to differences in the muscle fibre proportions.

\section{Lactate during exercise}

Significant differences were found between the sprinters and triathletes in the time course of their blood lactate concentrations. However, the peak values did not significantly differ between the triathletes and sprinters, although the triathletes continued their test at speeds of up to $18 \mathrm{~km} / \mathrm{h}$, while the sprinters only ran at up to $16 \mathrm{~km} / \mathrm{h}$.

In the triathletes, the increase in their blood lactate concentration was slow at the beginning of the test, but then increased rapidly near the end of the test. In contrast, the sprinters showed a faster increase in their blood lactate at lower treadmill speeds. Firstly, this may be due to the fact that the triathletes have a higher anaerobic threshold, which allows them to exercise at a higher relative intensity (percent of $\mathrm{VO}_{2 \max }$ ) for a longer time [13]. This means that more ATP is produced through oxidative phosphorylation and it does not need to be supplemented with lactate production-related anaerobic glycolysis [10]. Secondly, one might suppose that the late blood lactate accumulation in the triathletes may be due to a higher percentage of slow twitch (Type I) muscle fibres, which mainly rely on an aerobic metabolism to resynthesize ATP; however, this feature was not examined in this study. Thirdly, as MacRae et al. [15] noticed, in triathletes a slower lactate increase is caused by a faster lactate removal rate from the blood through oxidation. Lastly, the later blood lactate accumulation in triathletes could be caused by changes in their extracellular $\mathrm{pH}$. Banister et al. [2] suggested that a $\mathrm{pH}$ decrease is required for lactate to efflux from the muscle. Also, a significant increase in the blood ammonia, due to an impairment of ATP resynthesis, leads to a subsequent lactate accumulation.

\section{Recovery period}

This study was also undertaken with the aim of evaluating the post-exercise blood ammonia and blood lactate concentrations. In both groups, the concentration of ammonia and lactate in the blood did not achieve its resting value after 30 minutes of recovery. This data is in line with the findings of Ament et al. [1], who observed that lactate and ammonia concentrations in blood were still elevated after 30 minutes 
of recovery in healthy volunteers. Other research [4] indicates that lactate and ammonia appear to return to the baseline level within 24 hours; however, this was not analysed in our study. In our sprinters and triathletes, the blood ammonia and lactate levels decreased almost linearly during the recovery time. This suggests that in highly-trained athletes, both their speed-power and endurance training adaptations are equally efficient in supporting the removal of ammonia and lactate from the blood after exercise.

\section{Practical implications - lactate versus ammonia}

The blood ammonia accumulation was almost linear throughout the entire range of the exercise intensity; whereas the change in the blood lactate accumulation was nonlinear (slow at lower intensities and faster at higher intensities). This indicates that blood ammonia is a more sensitive indicator at lower exercise intensities than blood lactate. It therefore seems that blood ammonia may be a better marker for monitoring fatigue in long-duration aerobic exercise, since it increases proportionately to the exercise intensity even at low and moderate levels. Conversely, the exclusive use of lactate as an intensity indicator during the aerobic exercise does not provide sufficient information. At low intensities, the lactate concentration does not rise much above the baseline, which may be misinterpreted as the lack of an adequate exercise load. This is important for endurance-trained athletes (or for other athletes developing endurance to some degree), since much of their training volume is based on a moderate exercise intensity, which can stress the athlete considerably. However, lactate can still be used as a marker of muscle fatigue during anaerobic exercise, since its production is directly related to the anaerobic involvement in the ATP resynthesis. Thus, the best way to monitor exercise and training would be to use these two biomarkers simultaneously. Currently, this is even possible in field conditions (workout sessions), because portable and relatively cheap devices are manufactured for both ammonia and lactate assays.

\section{Strengths and limitations}

Some strengths of this study should be mentioned. Two groups of highly trained athletes practicing opposite sport disciplines were compared. Previous research has included physically active individuals but not competitive athletes [11, 18, 21]. Apart from the high level of sport performance, our sprinters and triathletes (all of whom are members of the Polish national team) were a very homogenous group with regards to their body build and composition, training status, training phase in the one-year cycle and, presumably, their genetic predispositions. Another strength of this study was the repeated measurement of the blood ammonia and lactate every 3 minutes during the incremental treadmill test, and throughout the 30 minutes of recovery. However, one limitation of this study is that the athletes were only examined during the preparatory phase of a one-year training cycle. Further research, performed during the competition and transition phases, is needed to show training-related adaptations and changes.

\section{Conclusion}

The study showed that the blood ammonia concentration may be a useful biomarker for exercise-related metabolic responses in competitive sprint-trained as well as in endurance-trained athletes. Blood ammonia proved to be more intensity-sensitive across the whole intensity range during an incremental exercise, especially at low intensity, whereas lactate did not differentiate the increase in exercise intensity. Therefore, ammonia should complement the use of classical biomarkers such as blood lactate concentration, in the training of high-level endurance athletes as well as speed-power athletes.

\section{References}

1. Ament W., J.R. Huizenga, E. Kort, T.W. van der Mark, R.G. Grevink, G.J. Verkerke (1999) Respiratory ammonia output and blood ammonia concentration during incremental exercise. Int. J. Sports Med., 20(2): 71-77. DOI: $10.1055 / \mathrm{s}-2007-971096$.

2. Banister E.W., M.E. Allen, I.B. Mekjavic, A.K. Singh, B. Legge, B.J.C. Mutch (1997) The time course of ammonia and lactate accumulation in blood during bicycle exercise. Eur. J. Appl. Physiol., 51: 195-202. DOI: 10.1007/BF00455182.

3. Billat V.L., A. Demarle, J. Slawinski, M. Paiva, J.P. Koralsztein (2001) Physical and training characteristics of the top-class marathon runners. Med. Sci. Sports Exerc., 33(12): 2089-2097.

4. Degoutte F., P. Jouanel, E. Filaire (2003) Energy demands during a judo match and recovery. Br. J. Sports Med., 37: 245-249. DOI: 10.1136/bjsm.37.3.245.

5. Dudley G.A., R.L. Terjung (1985) Influence of aerobic metabolism on IMP accumulation in fast-twitch muscle. Am. J. Physiol., 248: C37-C42.

6. Duffield R., B. Dawson (2003) Energy system contribution in track running. New Stud. Athlet., 18(4): 47-56.

7. Duffield R., B. Dawson, C. Goodman (2004) Energy system contribution to $100-\mathrm{m}$ and $200-\mathrm{m}$ track running events. J. Sci. Med. Sport, 7(3): 302-313. DOI: 10.1016/ S1440-2440(04)80025-2.

8. Duffield R., B. Dawson, C. Goodman (2005) Energy system contribution to 1500- and 3000-metre track running. J. Sports Sci., 23(10): 993-1002. DOI: 10.1080/02640410400021963.

9. Ferri A., S. Adamo, A. La Torre, M. Marzorati, D.J. Bishop, G. Miserocchi (2012) Determinants of performance in 
1,500-m runners. Eur. J. Appl. Physiol., 112: 3033-3043. DOI: $10.1007 / \mathrm{s} 00421-011-2251-2$.

10. Finsterer J. (2012) Biomarkers of peripheral muscle fatigue during exercise. BMC Musculoskelet. Disord., 13: 218. DOI: 10.1186/1471-2474-13-218.

11. Gorostiaga E.M., I. Navarro-Amézqueta, J.A.L. Calbet, L. Sánchez-Medina, R. Cusso, M. Guerrero, etal.(2014)Blood ammonia and lactate as markers of muscle metabolites during leg press exercise. J. Strength Cond. Res., 28(10): 2775-2785. DOI: 10.1519/JSC.0000000000000496.

12. Graham T.E., L.P. Turcotte, B. Kiens, E.A. Richter (1997) Effect of endurance training on ammonia and amino acid metabolism in humans. Med. Sci. Sports Exerc., 29: 646-653.

13. Green J.M., J.H. Hornsby, R.C. Pritchett, K. Pritchett (2014) Lactate threshold comparison in anaerobic vs aerobic athletes and untrained participants. Int. J. Exerc. Sci., 7(4): 329-338.

14. Hancock C.R., E. Janssen, R.L. Terjung (2006) Contraction-mediated phosphorylation of AMPK is lower in skeletal muscle of adenylate kinase-deficient mice. J. Appl. Physiol., 100: 406-413. DOI: 10.1152/ japplphysiol.00885.2005.

15. MacRae H.H., T.D. Noakes, S.C. Dennis (1995) Effects of endurance training on lactate removal by oxidation and gluconeogenesis during exercise. Pflugers Arch., 430(6): 964-970.

16. Mutch B.J., E.W. Banister (1983) Ammonia metabolism in exercise and fatigue: a review. Med. Sci. Sports Exerc., 15(1): 41-50.

17. Nybo L., M.K. Dalsgaard, A. Steensberg, K. Møller, N.H. Secher (2005) Cerebral ammonia uptake and accumulation during prolonged exercise in humans. J. Physiol., 563(Pt 1): 285-290. DOI: 10.1113/jphysiol.2004.075838.

18. Ogino K., T. Kinugawa, S. Osaki, M. Kato, A. Endoh, Y. Furuse, K. Uchida, M. Shimoyama, O. Igawa, I. Hisatome, C. Shigemasa (2000) Ammonia response to constant exercise: differences to the lactate response. Clin. Exp. Pharmacol. Physiol., 27(8): 612-617. DOI: 10.1046/j.1440-1681.2000.03312.x.

19. Philp A., A.L. Macdonald, P.W. Watt (2005) Lactate a signal coordinating cell and systemic function. J. Exp. Biol., 208: 4561-4575. DOI: 10.1242/jeb.01961.

20. Ravier G., B. Dugué, F. Grappe, J.D. Rouillon (2006) Maximal accumulated oxygen deficit and blood responses of ammonia, lactate and $\mathrm{pH}$ after anaerobic test: a comparison between international and national elite karate athletes. Int. J. Sports Med., 10: 810-817.

21. Sahlin K., M. Tonkonogi, K. Söderlund (1999) Plasma hypoxanthine and ammonia in humans during prolonged exercise. Eur. J. Appl. Physiol. Occup. Physiol., 80(5): 417-422.

22. Schumacker Y.O., P. Mueller (2002) The 4000-m team pursuit cycling world record: theoretical and practical aspects. Med. Sci. Sports Exerc., 34: 1029-1036. DOI: 10.1097/00005768-200206000-00020.
23. Snow R.J., M.F. Carey, C.G. Stathis, M.A. Febbraio, C.G. Hargreaves (2000) Effect of carbohydrate ingestion on ammonia metabolism during exercise in humans. J. Appl. Physiol., 88: 1576-1580.

24. Sola-Penna M. (2008) Metabolic regulation by lactate. IUBMB Life, 60(9): 605-608. DOI: 10.1002/iub.97.

25. Spencer M.R., P.B. Gastin (2001) Energy system contribution during 200- to 1500-m running in highly trained athletes. Med. Sci. Sports Exerc., 33(1): 157-162. DOI: 10.1097/00005768-20010100000024.

26. Terjung R.L, P.C. Tullson (1992) Ammonia metabolism during exercise. In: Lamb D.R. and C.V. Gisolfi (eds.) Energy Metabolism in Exercise and Sport. Dubuque, IA: Brown \& Benchmark, pp. 235-268.

27. Wilkinson D.J., N.J. Smeeton, P.W. Watt (2010) Ammonia metabolism, the brain and fatigue, revisiting the link. Prog. Neurobiol., 91: 200-219. DOI: 10.1016/j. pneurobio.2010.01.012.

28. Yuan Y., R. So, S. Wong, K.M. Chan (2002) Ammonia threshold - comparison to lactate threshold, correlation to other physiological parameters and response to training. Scand. J. Med. Sci. Sports, 12: 358-364. DOI: 10.1034/j.1600-0838.2002.00185.x.

29. Zieliński J., K. Kusy (2012) Training-induced adaptation in purine metabolism in high-level sprinters vs. triathletes. J. Appl. Physiol., 112: 542-551. DOI: 10.1152/ japplphysiol.01292.2011.

30. Zieliński J., K. Kusy (2015) Hypoxanthine: A universal metabolic indicator of the training status in competitive sport. Exerc. Sport Sci. Rev., 43(4): 214-221. DOI: 10.1249/JES.0000000000000055.

31. Zieliński J., B. Krasińska, K. Kusy (2013) Hypoxanthine as a predictor of performance in highly trained athletes. Int. J. Sports Med., 34(12): 1079-1086. DOI: 10.1055/s0033-1337947.

32. Zieliński J., K. Kusy, T. Rychlewski (2011) Effect of training load structure on purine metabolism in middle-distance runners. Med. Sci. Sports Exerc., 43(9): 1798-807. DOI: $10.1249 /$ MSS.0b013e318215d10b.

\section{Received 23.11.2015 \\ Accepted 20.03.2016}

(C) University of Physical Education, Warsaw, Poland

\section{Acknowledgments}

This work was funded by the Polish Ministry of Science and Higher Education from financial resources within "The Development of Academic Sports" programme (grant number RSA2 04152), and from financial resources of the National Science Centre within the OPUS 5 programme (grant number 2013/09/B/NZ7/02556). 